\title{
Diagonal Feature Extraction Based Handwritten Character System Using Neural Network
}

\author{
J.Pradeep \\ Department of ECE \\ Pondicherry Engineering College \\ Pondicherry, India
}

\author{
E.Srinivasan \\ Department of ECE \\ Pondicherry Engineering College \\ Pondicherry, India
}

\author{
S.Himavathi \\ Department of EEE \\ Pondicherry Engineering College \\ Pondicherry, India
}

\begin{abstract}
A handwritten character recognition system using multilayer Feed forward neural network is proposed in this paper. The character data set suitable for recognizing postal addresses contains 38 elements which include 26 alphabets, 10 numerals and 2 symbols. Fifteen different handwritten data sets were used for training the neural network for classification and recognition of the characters. Three different orientations, namely, horizontal, vertical and diagonal directions are used for extracting 54 features from each character. The trained neural recognition system is tested for various inputs and found to perform well. The diagonal orientation for feature extraction is identified to be the most suitable method as it yields higher recognition accuracy. The proposed system will aid applications for postal/parcel address recognition and conversion of any hand written document into structural text form.
\end{abstract}

\section{Keywords}

Handwritten character recognition, Image processing, Feature extraction, Feed forward neural networks.

\section{INTRODUCTION}

Handwriting Recognition has been one of the most fascinating and challenging research areas in field of image processing and pattern recognition in the recent years. It contributes immensely to the advancement of an automation process and can improve the interface between man and machine in numerous applications. Several research works have been focusing toward evolving newer techniques and methods that would reduce the processing time while providing higher recognition accuracy [1] [2].

In general, handwriting recognition is classified into two types as off-line and on-line handwriting recognition methods. In the off-line recognition, the writing is usually captured optically by a scanner and the completed writing is available as an image. But, in the on-line system the two dimensional coordinates of successive points are represented as a function of time and the order of strokes made by the writer are also available. The online methods have been shown to be superior to their off-line counterparts in recognizing handwritten characters due to the temporal information available with the former [3]. However, in the off-line systems, the neural networks have been successfully used to yield comparably high recognition accuracy levels [4]. Several applications including mail sorting, bank processing, document reading and postal address recognition require off-line handwriting recognition systems. As a result, the off-line handwriting recognition continues to be an active area for research towards exploring the newer techniques that would improve recognition accuracy [5] [6].

The first important step in any handwritten recognition system is preprocessing followed by segmentation and feature extraction. Preprocessing includes the steps that are required to shape the input image into a form suitable for segmentation [7]. In the segmentation, the input image is segmented into individual characters and then, each character is resized into $\mathrm{m} \times \mathrm{n}$ pixels towards the extracting the features.

The Selection of appropriate feature extraction method is probably the single most important factor in achieving high recognition performance. Several methods of feature extraction for character recognition have been reported in the literature [8]. The widely used feature extraction methods are Template matching, Deformable templates, Unitary Image transforms, Graph description, Projection Histograms, Contour profiles, Zoning, Geometric moment invariants, Zernike Moments, Spline curve approximation, Fourier descriptors, Gradient feature and Gabor features.

An artificial neural Network as the backend is used for performing classification and recognition tasks. In the off-line recognition system, the neural networks have emerged as the fast and reliable tools for classification towards achieving high recognition accuracy [9].

In this paper, a diagonal feature extraction scheme for the recognizing off-line handwritten characters is proposed. In the feature extraction process, resized individual character of size $90 x 60$ pixels is further divided into 54 equal zones, each of size $10 \times 10$ pixels. The features are extracted from the pixels of each zone by moving along its diagonals. This procedure is repeated for all the zones leading to extraction of 54 features for each character. These extracted features are used to train a feed forward back propagation neural network employed for performing classification and recognition tasks. Extensive simulation studies show that the recognition system using diagonal features provides good recognition accuracy.

The paper is organized as follows. In section 2, the proposed recognition system is presented. The feature extraction procedure adopted in the system is detailed in the section 3 . Section 4 describes the classification and recognition using feed forward back propagation neural network. Section 5. presents the experimental results and comparative analysis. In Section 6, the proposed recognition system in Graphical User Interface is presented and finally, the paper is concluded in section 7 . 


\section{THE PROPOSED RECODGNITION SYSTEM}

A typical handwriting recognition system consists of preprocessing, segmentation, feature extraction, classification and recognition, and post processing stages. The schematic diagram of the proposed recognition system is shown in Figure.1

\subsection{Image Acquisition}

In Image acquisition, the recognition system acquires a scanned image as an input image. The image should have a specific format such as JPEG, BMT etc. This image is acquired through a scanner, digital camera or any other suitable digital input device.

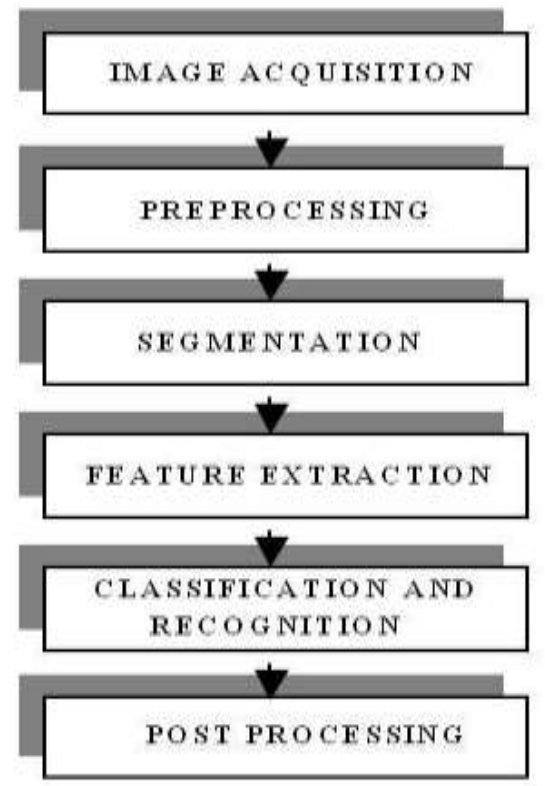

Figure 1. Schematic diagram of the proposed off-line recognition system

\subsection{Preprocessing}

The preprocessing is a series of operations performed on the scanned input image. It essentially enhances the image rendering it suitable for segmentation. The various tasks performed on the image in preprocessing stage are shown in Figure.2. Binarization process converts a gray scale image into a binary image using global thresholding technique. Detection of edges in the binarized image using sobel technique, dilation the image and filling the holes present in it are the operations performed in the last two stages to produce the preprocessed image [10] suitable for segmentation.

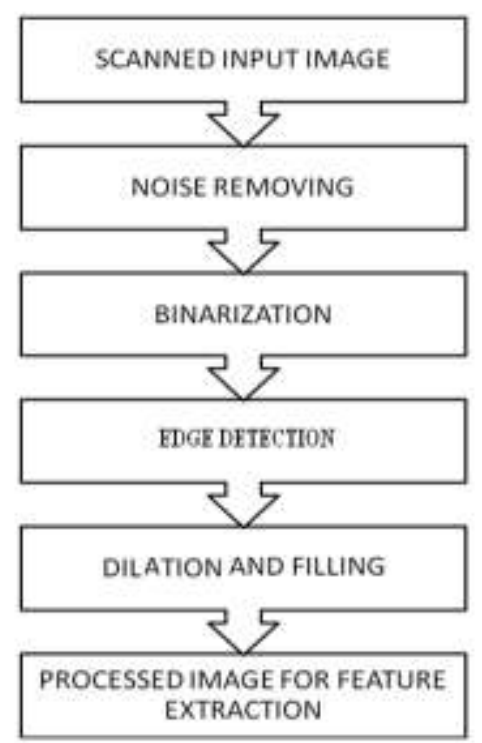

Figure 2. Preprocessing of handwritten character of image

\subsection{Segmentation}

In the segmentation stage, an image of sequence of characters is decomposed into sub-images of individual character [11]. In the proposed system, the preprocessed input image is segmented into isolated characters by assigning a number to each character using a labeling process. This labeling provides information about number of characters in the image. Each individual character is uniformly resized into 90X60 pixels for extracting its features.

\section{FEATURE EXTRACTION}

In this stage, the features of the characters that are crucial for classifying them at recognition stage are extracted. This is an important stage as its effective functioning improves the recognition rate and reduces the misclassification [12]. Diagonal feature extraction scheme for recognizing off-line handwritten characters is proposed in the work. Every character image of size 90x 60 pixels is divided into 54 equal zones, each of size 10x10 pixels (Figure.3(c)). The features are extracted from each zone pixels by moving along the diagonals of its respective $10 \mathrm{X} 10$ pixels. Each zone has 19 diagonal lines and the foreground pixels present along each diagonal line is summed to get a single subfeature and thus 19 sub-features are obtained from the each zone. These 19 sub-features values are averaged to form a single feature value and placed in the corresponding zone (Figure. 3 (b)). This procedure is sequentially repeated for the all the zones. There could be some zones whose diagonals are empty of foreground pixels. The feature value corresponding to these zones are zero. Finally, 54 features are extracted for each character. In addition, 9 and 6 features are obtained by averaging the values placed in zones rowwise and columnwise, respectively. As a result; every character is represented by 69 , that is, $54+15$ features. 


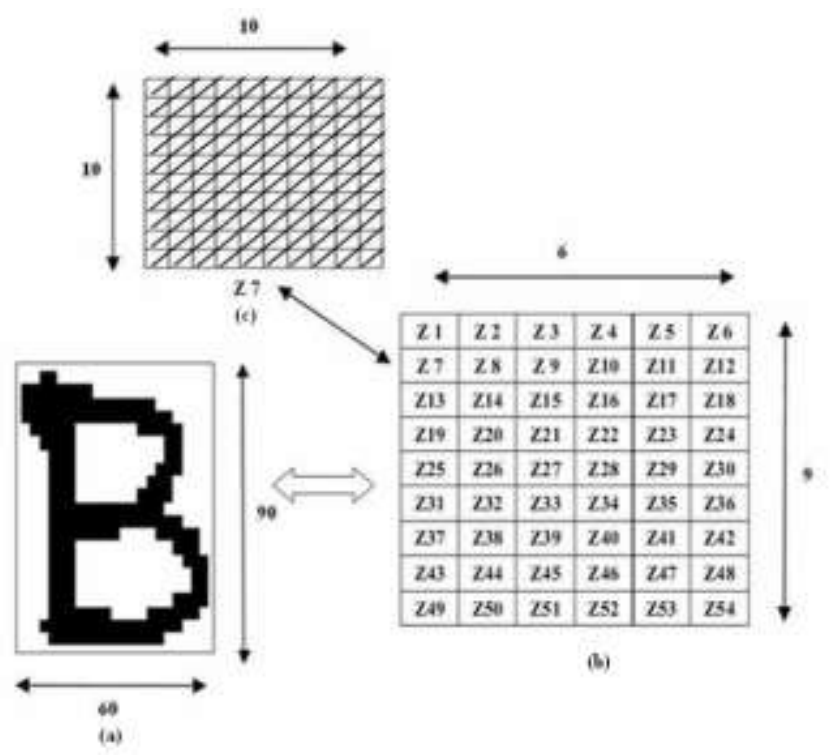

Figure 3. Feature Extraction

\section{CLASSIFICATION AND RECOGNITION}

The classification stage is the decision making part of a recognition system and it uses the features extracted in the previous stage. A feed forward back propagation neural network having two hidden layers with architecture of 54-100-100-38 is used to perform the classification. The hidden layers use log sigmoid activation function, and the output layer is a competitive layer as one of the characters have to be identified. The feature vector is denoted as $\mathrm{X}$, and defined as $X=\left(f_{1}, f_{2}, \ldots, f_{d}\right)$, where $f$ denotes features and $d$ is the number of zones into which each character is divided. The number of input neurons is determined by length of the feature vector $d$. The total numbers of characters $n$ determines the number of neurons in the output layer. The number of neurons in each hidden layer is obtained by trial and error.

The architecture of the network having two layers is illustrated in Figure. 4. The output of $i^{\text {th }}$ layer is given by

$$
a^{i}=\log \operatorname{sig}\left(w^{i} a^{i-1}+b^{i}\right)
$$

where,

$$
\begin{aligned}
i & =[1,2,3] \text { and } a^{0}=\mathbf{P} \\
w^{i} & =\text { weight vector of } i^{\text {th }} \text { layer } \\
a^{i} & =\text { output of } i^{\text {th }} \text { layer } \\
b^{i} & =\text { bias vector for } i^{\text {th }} \text { layer }
\end{aligned}
$$

The network training parameters are:

$>$ Input nodes : $54 / 69$

$>$ Hidden nodes : 100 each

$>$ Output nodes :38 (26 alphabets, 10 numerals and 2 special symbols)

$>$ Training algorithm : Gradient descent with momentum training and adaptive learning

$>$ Perform function :Mean Square Error

$>$ Training goal achieved : 0.000001

$>$ Training epochs :1000000

$>$ Training momentum constant : 0.9
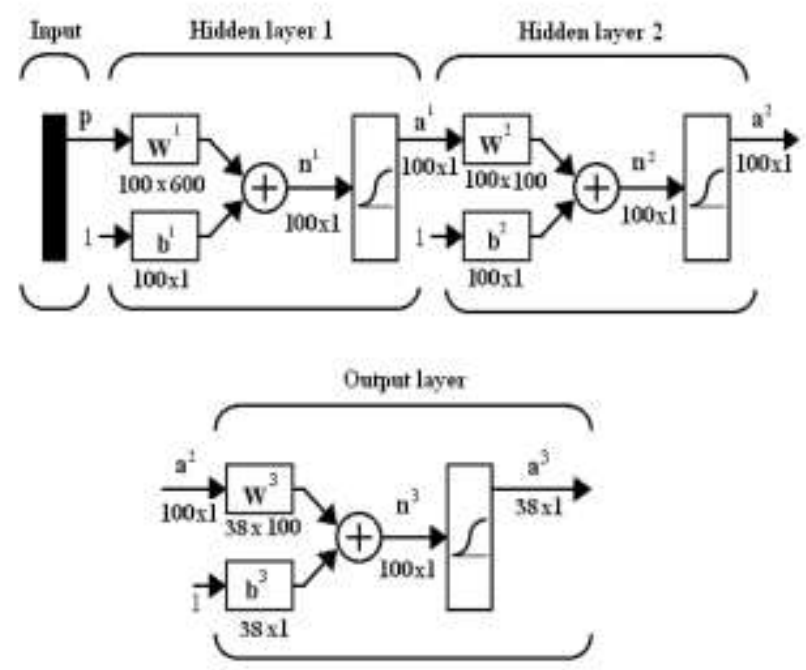

Figure 4. Three layer neural network for character recognition.

\section{EXPERIMENTAL RESULTS}

The recognition system has been implemented using Matlab7.1.The scanned image is taken as dataset/ input and feed forward architecture is used. The structure of neural network includes an input layer, two hidden layers each with 100 neurons and an output layer with 38 neurons. The gradient descent back propagation method with momentum and adaptive learning rate and log-sigmoid transfer functions are used for neural network training. Neural network has been trained using known dataset. Recognition systems using 64 and 59 features are built. The number of input nodes is chosen based on the number of features. After adequately training the network [13], the recognition system was tested using several unknown datasets and the results obtained are presented in this section. The training and testing data set image are shown in Figure. 5 and Figure .6.

Two approaches with three different ways of feature extraction are used for character recognition in the proposed system. The three different ways of feature extraction are horizontal direction, vertical direction and diagonal direction.

In the first approach, the feature vector size is chosen as 54, i.e. without rowwise and columnwise features. The results obtained using three different types of feature extraction are summarized 
in Table 1. The criteria for choosing the type of feature extraction are the speed of convergence, i.e. small number of epochs required to achieve the training goal and training stability. However, the most important parameter of interest is the accuracy of the recognition system. The results presented in Table 1 show that the diagonal feature extraction yields higher recognition accuracy compared to the others types of feature extraction.

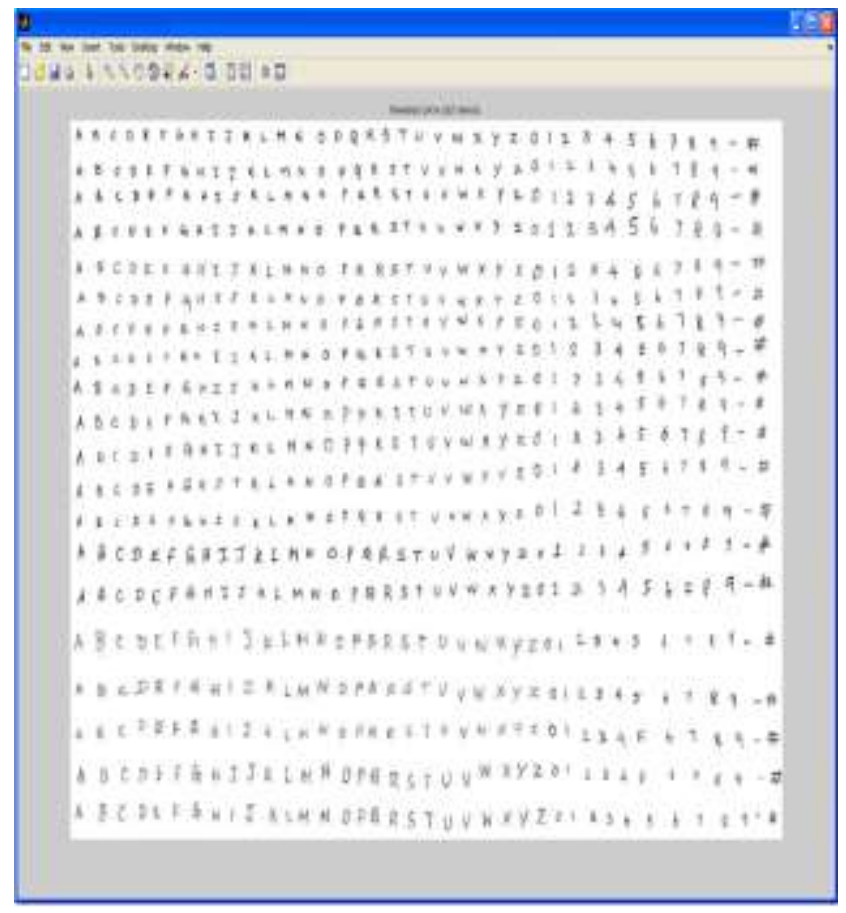

Figure 5. Training data set image

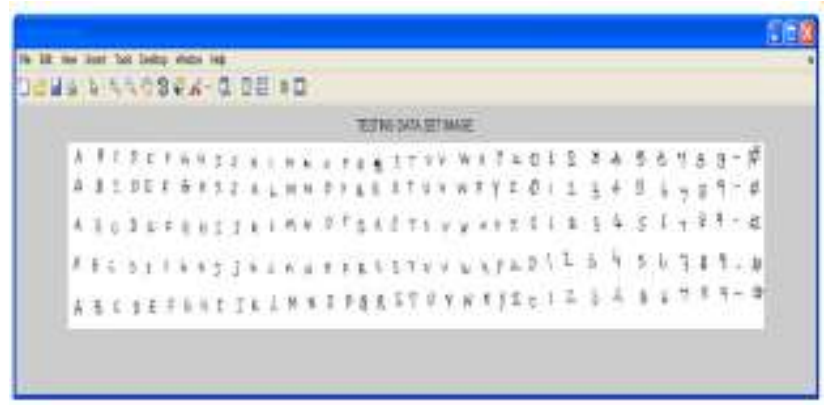

Figure 6. Testing data set image

In the second approach, the feature vector size is chosen as 69 including the rowwise and columnwise features. Three types of feature extraction are used for extracting the features and the results obtained are given in Table.2. It can be seen from Table. 2 that the diagonal feature extraction provides for better recognition accuracy than the other types of feature extraction.

Table 1. Recognition rate results obtained with 54 features

Networks

I

II

III

\begin{tabular}{|l|c|c|c|}
\hline $\begin{array}{l}\text { Feature Extraction } \\
\text { type }\end{array}$ & Vertical & Horizontal & Diagonal \\
\hline $\begin{array}{l}\text { Number of nodes in } \\
\text { input layer }\end{array}$ & 54 & 54 & 54 \\
\hline $\begin{array}{l}\text { Number of nodes in } \\
1 \text { st hidden layer }\end{array}$ & 100 & 100 & 100 \\
\hline $\begin{array}{l}\text { Number of nodes in } \\
2 \text { st hidden layer }\end{array}$ & 100 & 100 & 100 \\
\hline $\begin{array}{l}\text { Number of nodes in } \\
\text { output layer }\end{array}$ & 38 & 38 & 38 \\
\hline $\begin{array}{l}\text { No of training } \\
\text { elements }\end{array}$ & 570 & 570 & 570 \\
\hline $\begin{array}{l}\text { No of testing } \\
\text { elements }\end{array}$ & 80.5 & 84.21 & 98 \\
\hline $\begin{array}{l}\text { Recognition rate } \\
\text { percentage }\end{array}$ & 190 & & 190 \\
\hline
\end{tabular}

Table 2. Recognition rate results obtained with 69 features

\begin{tabular}{|l|c|c|c|}
\hline \multicolumn{1}{|c|}{ Networks } & I & II & III \\
\hline $\begin{array}{l}\text { Feature Extraction } \\
\text { type }\end{array}$ & Vertical & Horizontal & Diagonal \\
\hline $\begin{array}{l}\text { Number of nodes in } \\
\text { input layer }\end{array}$ & 69 & 69 & 69 \\
\hline $\begin{array}{l}\text { Number of nodes in } \\
\text { 1st hidden layer }\end{array}$ & 100 & 100 & 100 \\
\hline $\begin{array}{l}\text { Number of nodes in } \\
\text { 2st hidden layer }\end{array}$ & 100 & 100 & 100 \\
\hline $\begin{array}{l}\text { Number of nodes in } \\
\text { output layer }\end{array}$ & 38 & 38 & 38 \\
\hline $\begin{array}{l}\text { No of training } \\
\text { elements }\end{array}$ & 570 & 570 & 570 \\
\hline $\begin{array}{l}\text { No of testing } \\
\text { elements }\end{array}$ & 190 & 190 & 190 \\
\hline $\begin{array}{l}\text { Recognition rate } \\
\text { percentage }\end{array}$ & 85.26 & 96.31 & 99 \\
\hline
\end{tabular}

\section{IMPLEMENTATION ON GRAPHICAL USER INTERFACE}

A user-friendly front end interface as shown in Fig.7 has been implemented for the proposed handwritten character recognition system using MATLAB GUI (Graphical User Interface) [14]. The interface menu presents the user with five processing stages, three displays and two selection keys. The GUI enables the user to perform preprocessing, select the type of feature extraction, perform the feature extraction using the chosen method and train the network. After the network is trained, the test image is chosen using the facility provided for selecting the test images. The recognition of the test image can be initiated by clicking the recognition bar on the interface. The recognized image appears on the screen as shown in Fig.7. The same procedure can be used 
to select and recognize any number of test images. Finally the exit bar is used to quit from the character recognition system after recognizing all the test images. The GUI frees the user from the difficulties of working from the command line interface.

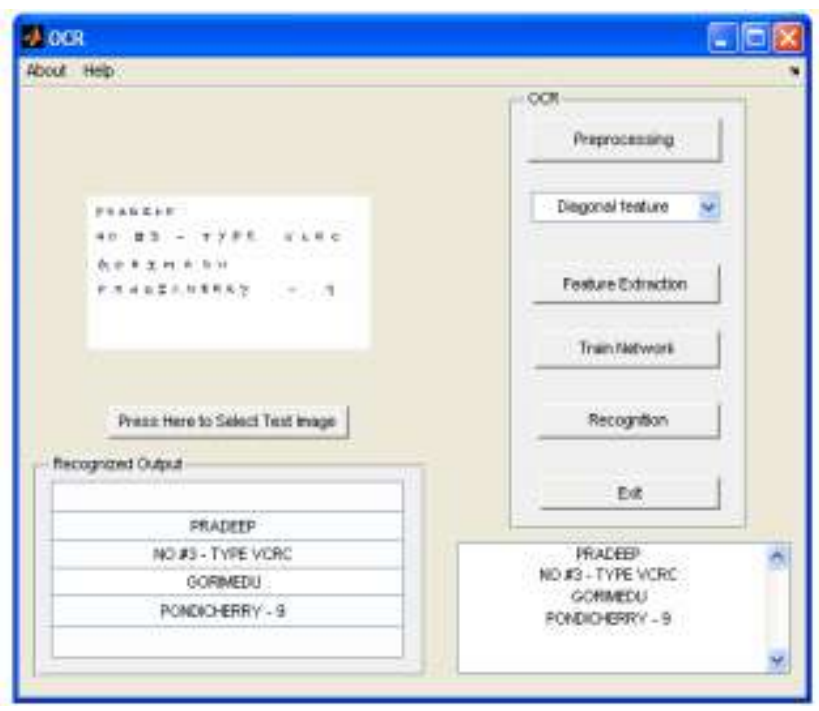

Figure 7. Handwritten character recognition system in Graphical User Interface

\section{CONCLUSION}

A simple and an efficient off-line handwritten character recognition system using a new type of feature extraction, namely, diagonal feature extraction is proposed. Two approaches using 54 features and 69 features are chosen to build the Neural Network recognition system. To compare the recognition efficiency of the proposed diagonal method of feature extraction, the Neural network recognition system is trained using the horizontal and vertical feature extraction methods. Six different recognition networks are built. It is shown that 69 features gives better recognition accuracy than 54 features for all the types of feature extraction. From the test results it is identified that the diagonal method of feature extraction yields the highest recognition accuracy of $98 \%$ for 54 features and 99\% for 69 features. The superiority of diagonal method of feature extraction is verified using a number of test images. The proposed off-line hand written character recognition system with superior recognition rates will be eminently suitable for serval applications including postal/parcel address recognition, bank proecssing, document reading and conversion of any handwritten document into structural text form.

\section{ACKNOWLEDGMENTS}

The authors duly acknowledge Mr.N.M.Prakash, B.Tech graduate of Pondicherry Engineering College, Puducherry for his contributive support towards completing this research work.

\section{REFERENCES}

[1] S. Mori, C.Y. Suen and K. Kamamoto, "Historical review of OCR research and development," Proc. of IEEE, vol. 80, pp. 1029-1058, July 1992.

[2] V.K. Govindan and A.P. Shivaprasad, "Character Recognition - A review," Pattern Recognition, vol. 23, no. 7, pp. 671- 683, 1990.

[3] R. Plamondon and S. N. Srihari, "On-line and off- line handwritten character recognition: A comprehensive survey,"IEEE. Transactions on Pattern Analysis and Machine Intelligence, vol. 22, no. 1, pp. 63-84, 2000

[4] U. Bhattacharya, and B. B. Chaudhuri, "Handwritten numeral databases of Indian scripts and multistage recognition of mixed numerals," IEEE Transaction on Pattern analysis and machine intelligence, vol.31, No.3, pp.444-457, 2009.

[5] U. Pal, T. Wakabayashi and F. Kimura, "Handwritten numeral recognition of six popular scripts," Ninth International conference on Document Analysis and Recognition ICDAR 07, Vol.2, pp.749-753, 2007.

[6] Pal, U. and B.B. Chaudhuri, "Indian script character recognition: A survey," Pattern Recognition, vol. 37, no.9, pp. 1887-1899, 2004.

[7] Mantas, J, "An overview of character recognition methodologies," Pattern Recognition, Vol.19,Issue 6, pp. 425-430, 1986.

[8] Anil.K.Jain and Torfinn Taxt, "Feature extraction methods for character recognition-A Survey," Pattern Recognition, vol. 29, no. 4, pp. 641-662, 1996.

[9] M. Hanmandlu, K.R.M. Mohan, and H. Kumar, "Neuralbased Handwritten character recognition," in Proceedings of Fifth IEEE International Conference on Document Analysis and Recognition, ICDAR'99, pp. 241-244, Bangalore, India, 1999.

[10] Rafael C. Gonzalez, Richard E. woods and Steven L.Eddins, Digital Image Processing using MATLAB, Pearson Education, Dorling Kindersley, South Asia, 2004.

[11] R.G. Casey and E.Lecolinet, "A Survey of Methods and Strategies in Character Segmentation," IEEE Transactions on Pattern Analysis and Machine Intelligence, Vol. 18, No.7, July 1996, pp. 690-706.

[12] S.V. Rajashekararadhya, and P.Vanajaranjan, "Efficient zone based feature extraction algorithm for handwritten numeral recognition of four popular south-Indian scripts," Journal of Theoretical and Applied Information Technology, JATIT vol.4, no.12, pp.1171-1181, 2008.

[13] J. Stephen Chapman, MATLAB Programming for Engineers, 2nd Edition, Brooks Kole Publishing Company, 2002.

[14] Lim Zong Ren "Thesis on Recognition of Handwriting on Postal Letters and parcels," Sim university, 2009. http://sst.unisim.edu.sg:8080/dspace/bitstream/123456789/1 87/1/09_Lim\%20Zong\%20Ren.pdf. 


\section{AUTHORS:}

J. Pradeep received his B.Tech degree in Electronics and Communication Engineering from Barathiyar college of Engineering and Technogy affiliated to Pondicherry University in the year 2005. He obtained his M.Tech degree in Electronics and Communication Engineering from Podicherry Engineering College in the year 2009. He is currently a Ph.D candidate in the Department of Electronics and Communication Engineering in Podicherry Engineering College. He has published a paper in International Journal. He is a life member of ISTE. His areas of interest are Wireless Communication, Image proceesing and Neural networks.

E. Srinivasan obtained his B.E. degree in Electrical and Electronics Engineering from P.S.G. College of Technology, Coimbatore, India, in the year 1984. He received his M.E. degree in Instrumentation Technology in the year 1987 from Madras Institute of Technology, Chennai, India. He was awarded with Ph.D. degree by the Anna University, Chennai, India in the year 2003 for his research contributions in Nonlinear Signal Processing. Currently, he is serving as Professor and Head of the
Department of Electronics and Communication Engineering, Pondicherry Engineering College, Pondicherry, India. He has published 30 research papers in national/international journals/conferences. He is a reviewer of the AMSE journal of Signal Processing. His research interests include nonlinear signal processing and pattern recognition and their applications.

S. Himavathi completed her BE degree in Electrical and Electronics Engineering from College of Engineering, Guindy, Chennai, India, in the year 1984. She obtained her M.E. degree in Instrumentation Technology in the year 1987 from Madras Institute of Technology, Chennai, India. She completed her Ph.D. degree in the area of Fuzzy modeling in the year 2003 from Anna University, Chennai, India. She is a Professor and Head of the Department of Electrical and Electronics Engineering, Pondicherry Engineering College. She has around 50 publications to her credit. She is a reviewer of the AMSE journal of Modeling, IEEE Industrial Electronics Society and Asian Neural Networks Society. Her research interests are Fuzzy systems, Neural Networks, Hybrid systems and their applications. 